\title{
Enantioselective Synthesis of 4-Silyl-1,2,3,4-tetrahydroquinolines via Copper(I) Hydride Catalyzed Asymmetric Hydrosilylation of 1,2-Dihydroquinolines
}

\author{
Qing-Feng Xu-Xu \\ Pusu Yang \\ Xiao Zhang
}

Shu-Li You*(i)

State Key Laboratory of Organometallic Chemistry, Center for Excellence in Molecular Synthesis, Shanghai Institute of Organic Chemistry, Chinese Academy of Sciences, 345 Lingling Lu, Shanghai 200032, P. R. of China

slyou@sioc.ac.cn

Dedicated to Prof. Barry M. Trost

Published as part of the Cluster The Power of Transition Metals: An Unending Well-Spring of New Reactivity

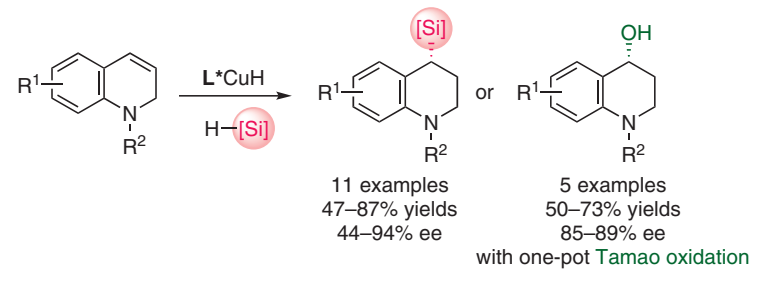

explored and most of the reported studies are restricted to the modification of amino acids. ${ }^{9}$ Despite the considerable progress, incorporation of silicon moieties on diverse bioactive scaffolds is still in great demand.

1,2,3,4-Tetrahydroquinolines (THQs) represent an important bioactive skeleton because of their ubiquitous presence in pharmaceuticals and natural products. ${ }^{10}$ Diverse methods have been developed for the synthesis of chiral THQs such as assorted cyclizations (e.g., Povarov reaction, ${ }^{11}$ Michael addition, ${ }^{12} \mathrm{C}-\mathrm{H}$ bond functionalization $\left.{ }^{13}\right),{ }^{14}$ kinetic resolution ${ }^{15}$ and dearomatization. In particular, asymmetric dearomatization of quinolines constitutes a straightforward approach. By utilizing (transfer) hydrogenation ${ }^{16}$ or the Reissert type reaction, ${ }^{17}$ various enantioenriched THQs were obtained from readily available quinolines. Recently, a stepwise reduction of quinolines and asymmetric catalytic transformation of the generated dihydroquinolines emerged as an attractive method to access chiral THQs with no need for preactivation of quinoline substrates. ${ }^{18}$ Thus the combination of this strategy with the introduction of silyl groups would be an appealing approach for the synthesis of silyl substituted THQs. ${ }^{19}$ Compared with other methods of forging $\mathrm{C}\left(\mathrm{sp}^{3}\right)$-Si bonds, ${ }^{20}$ transition-metal-catalyzed asymmetric hydrosilylation of unsaturated compounds ${ }^{21}$ represents a direct and atom-economic approach. ${ }^{22}$ In this regard, we envisioned that copper-catalyzed asymmetric hydrosilylation of 1,2-dihydroquinolines would efficiently introduce a C-Si bond at the 4-position of THQs. ${ }^{23}$ Herein, we report the results of this study.

At the outset, $\mathrm{N}-\mathrm{CO}_{2} \mathrm{Me}$ 1,2-dihydroquinoline (1a) was chosen as the substrate in the hydrosilylation reaction (Table 1). Considering the relatively high reactivity of arylsilanes over alkylsilanes, we chose diphenylsilane for the initial 


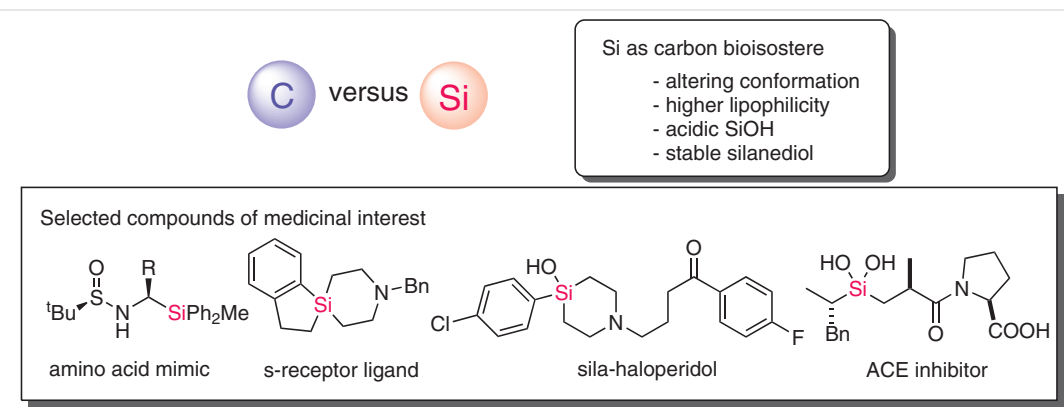

Access to silicon-containing potential bioactive compounds
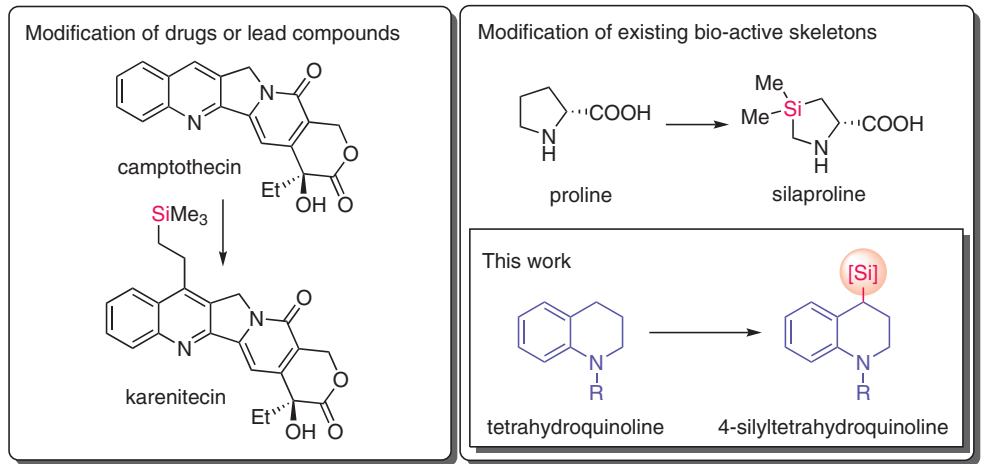

Scheme 1 Application of silicon in pharmaceutical chemistry

attempt. Compound 1a was treated with 3 equivalents of diphenylsilane in the presence of the catalyst derived from copper(II) acetate and $\mathbf{L 1}$ at $40^{\circ}$ under neat conditions for 36 h. To our delight, the desired product 2a was afforded in $56 \%$ yield and $87 \%$ ee (entry 1 ). Notably, the addition of a mono-phosphine as the secondary ligand ${ }^{24}$ resulted in an improved yield (entries 2-5; see the Supporting Information for more details), and ( $p$-tolyl $)_{3} \mathrm{P}$ gave the optimal results ( $86 \%$ yield and $87 \%$ ee; entry 4$).{ }^{25}$ Subsequent screening of chiral ligands revealed that $\mathbf{L 1}$ is the most effective ligand (entries 6-11).

With the optimal conditions in hand, the substrate scope was then explored (Scheme 2; see also the Supporting Information and Procedure A). ${ }^{26}$ The substrates with various $\mathrm{N}$-protecting groups $\left(\mathrm{Ac}, \mathrm{CO}_{2} \mathrm{Bn}, \mathrm{CO}_{2}{ }^{i} \mathrm{Bu}\right.$ ) gave the desired products with good yields and enantioselectivities (2b-d, 74-83\% yields, 83-90\% ee). A series of substituents at the 6-position of 1,2-dihydroquinolines were explored. A moderate yield and poor enantioselectivity were observed for the bromo-bearing substrate (2e, $50 \%$ yield, $46 \%$ ee). The substrate with an electron-withdrawing group $\left(\mathrm{CO}_{2} \mathrm{Me}\right)$ worked well, affording the desired THQ in $87 \%$ yield and 89\% ee (2f). 6-Thienyl-1,2-dihydroquinoline was transformed into its corresponding product $\mathbf{2} \mathbf{g}$ in moderate yield with slightly decreased ee value (47\% yield, $76 \%$ ee). The THQs with electron-donating groups (OMe and SMe) were obtained with good results (2h, $87 \%$ yield, $94 \%$ ee; $\mathbf{2 i}, 82 \%$ yield, $86 \%$ ee). Substituent effects were also investigated for the 7-methyl (2j) and 7-methoxy (2k) substrates, giving $77 \%$ yield with $82 \%$ ee and $62 \%$ yield with $82 \%$ ee, respectively. Subsequently, by utilizing phenylsilane as the silyl reagent, the desired 4-silyl THQs were generated and an extra Tamao oxidation was performed in a one-pot fashion, yielding 4-hydroxy THQs (Scheme 3; see also the Supporting Information and Procedure B). ${ }^{27}$ The $\mathrm{N}-\mathrm{CO}_{2} \mathrm{Me}$ and $\mathrm{N}-\mathrm{CO}_{2} \mathrm{Bn}$ 1,2-dihydroquinolines were well tolerated, leading to the desired products $\mathbf{3} \mathbf{a}^{28}$ and $\mathbf{3 b}$ in $63 \%$ yield with $89 \%$ ee and $73 \%$ yield with $88 \%$ ee, respectively. 1,2-Dihydroquinolines bearing varied substituents (6-OMe, 6-Ph and 7-Me) reacted smoothly with phenylsilane, and moderate yields with good enantioselectivities were obtained (3c-e, 50-68\% yields, 85-89\% ee). Notably, other silanes such as $\mathrm{Et}_{2} \mathrm{SiH}_{2}$ and $\mathrm{Et}_{2} \mathrm{MeSiH}$ were also tested, but failed to give any desired product.

To demonstrate the practicality of this protocol, we next performed scale-up reactions (Scheme 4). The hydrosilylation of 1a with diphenylsilane at $5 \mathrm{mmol}$ scale under the standard conditions gave an improved yield and slightly decreased enantioselectivity (eq. 1, 1.76 g, 94\% yield, 85\% ee). The one-pot hydrosilylation/Tamao oxidation reaction occurred smoothly with $2.5 \mathrm{~mol} \%$ catalyst loading (eq. 2, 168 $\mathrm{mg}, 81 \%$ yield, $89 \%$ ee). The 4 -silyl THQ product $2 \mathrm{a}$ could be oxidized to the desired silanol in $83 \%$ yield with $85 \%$ ee (Scheme 5). The absolute configuration of 4 was determined to be $R$ by the X-ray crystallographic analysis of its optically pure single crystal and the absolute configurations 
Table 1 Optimization of the Reaction Conditions ${ }^{\mathrm{a}}$

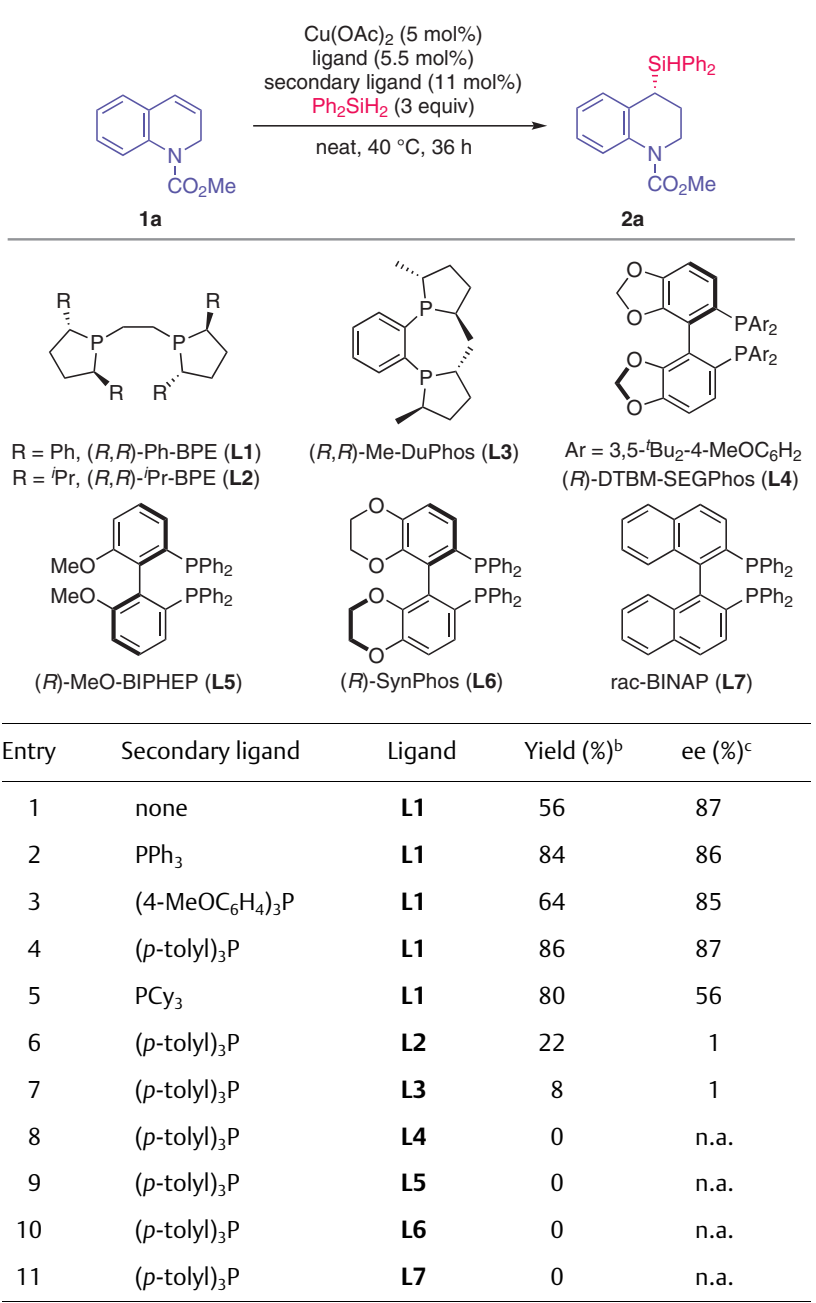

a Reaction conditions: $\mathrm{Cu}(\mathrm{OAc})_{2}(0.010 \mathrm{mmol})$, ligand $(0.011 \mathrm{mmol})$, secondary ligand ( $0.022 \mathrm{mmol}$, if used), $1 \mathrm{a}(0.2 \mathrm{mmol})$ and diphenylsilane (0.6 $\mathrm{mmol}$ ) were stirred at $40^{\circ} \mathrm{C}$ under neat conditions for $36 \mathrm{~h}$.

b Isolated yield.

' The ee value was determined based on HPLC analysis; n.a. = not applicable.

of the products $\mathbf{2}$ and $\mathbf{3}$ were assigned based on the assignment of $4 .^{29}$

A deuterium experiment utilizing $\mathrm{Ph}_{2} \mathrm{SiD}_{2}$ as the hydride source revealed that hydrogen atoms at both the 3position and in the silyl group were deuterated, which demonstrated the excellent atom-economy of this protocol (Scheme 6). A plausible mechanism was thus proposed as exemplified by the reaction between 1a and diphenylsilane (Scheme 7). Ligated copper hydride $(\mathbf{L C u H})$ is generated in situ from the copper(II) acetate, the ligand (L) and diphenylsilane. The activated species then inserts into 1a with formation of intermediate $\mathbf{A}$ containing the stereogenic center with a $\mathrm{C}-\mathrm{Cu}$ bond. Subsequent stereoretentive $\sigma$ metathesis between $\mathbf{A}$ and another silane molecule results in the desired product $\mathbf{2 a}$ and the regeneration of $\mathbf{L C u H} .{ }^{30}$

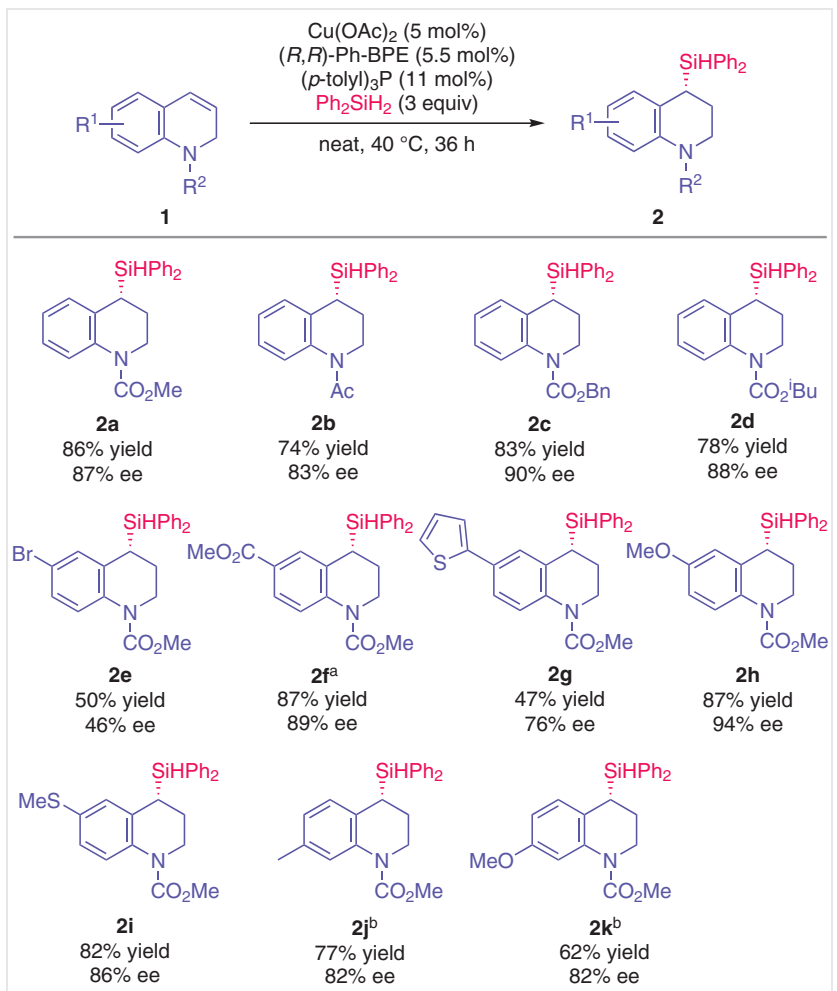

Scheme 2 Substrate scope for the asymmetric hydrosilylation. $R e$ agents and conditions: $\mathrm{Cu}(\mathrm{OAc})_{2}(0.010 \mathrm{mmol}),(R, R)-\mathrm{Ph}-\mathrm{BPE}(0.011$ mmol), ( $p$-tolyl) $)_{3} \mathrm{P}(0.022 \mathrm{mmol}), 1(0.2 \mathrm{mmol})$ and diphenylsilane $(0.6$ $\mathrm{mmol}$ ) were stirred at $40{ }^{\circ} \mathrm{C}$ under neat condition for $36 \mathrm{~h}$. Isolated yields are reported and the ee value was determined based on HPLC or SFC analysis. ${ }^{\text {a }} 5$ equiv of silane were used. ${ }^{\text {b }}$ The reaction time was $48 \mathrm{~h}$.

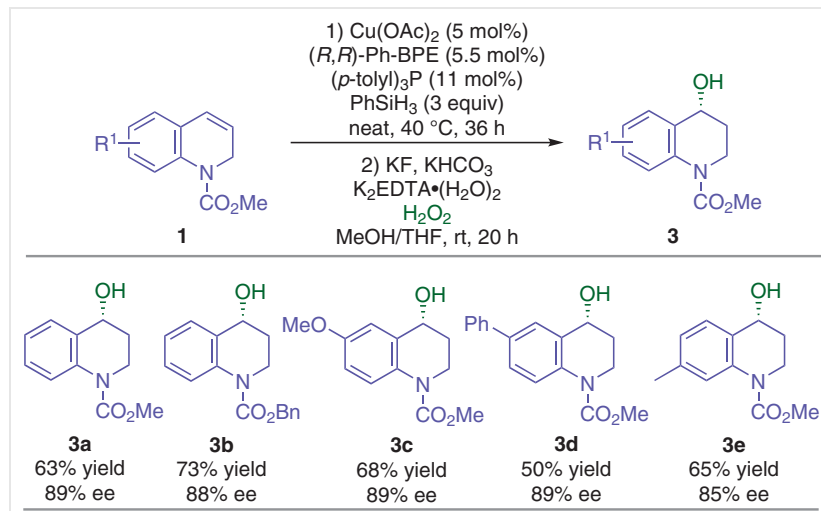

Scheme 3 Substrate scope for the asymmetric hydrosilylation and Tamao oxidation. Reagents and conditions: $\mathrm{Cu}(\mathrm{OAc})_{2}(0.010 \mathrm{mmol})$, (R,R)-Ph-BPE (0.011 mmol), ( $p$-tolyl) ${ }_{3} \mathrm{P}(0.022 \mathrm{mmol}), 1$ (0.2 mmol) and phenylsilane $(0.6 \mathrm{mmol})$ were stirred at $40{ }^{\circ} \mathrm{C}$ under neat conditions for $36 \mathrm{~h}$. Then $\mathrm{KF}(0.8 \mathrm{mmol}), \mathrm{KHCO}_{3}(0.8 \mathrm{mmol}), \mathrm{K}_{2} \mathrm{EDTA} \cdot\left(\mathrm{H}_{2} \mathrm{O}\right)_{2}(0.2$ $\mathrm{mmol}), \mathrm{MeOH}(1.2 \mathrm{~mL})$ and $\mathrm{H}_{2} \mathrm{O}_{2}(1.8 \mathrm{mmol})$ were added and stirred at r.t. in THF (1.2 mL) for $20 \mathrm{~h}$. Isolated yields are given and the ee values were determined based on HPLC or SFC analysis. 


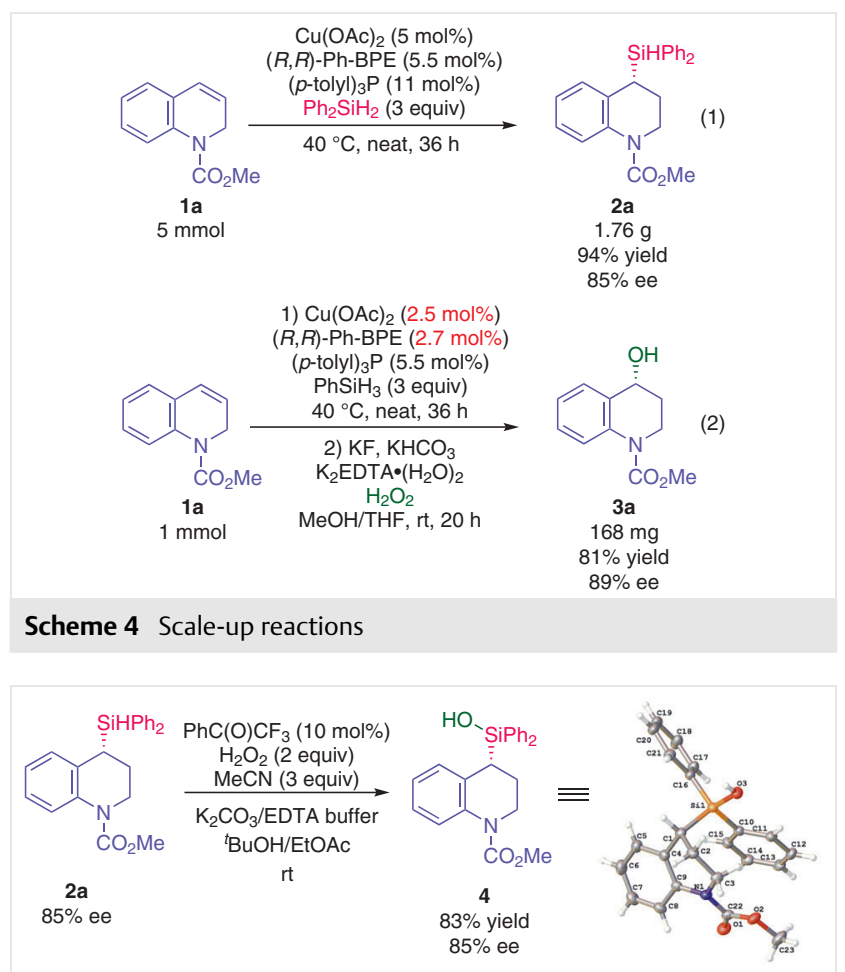

Scheme 5 Transformation into product 4

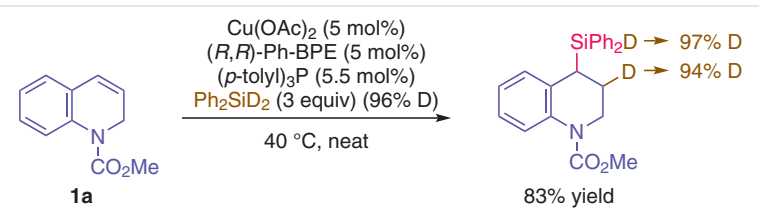

Scheme 6 Deuterium experiment

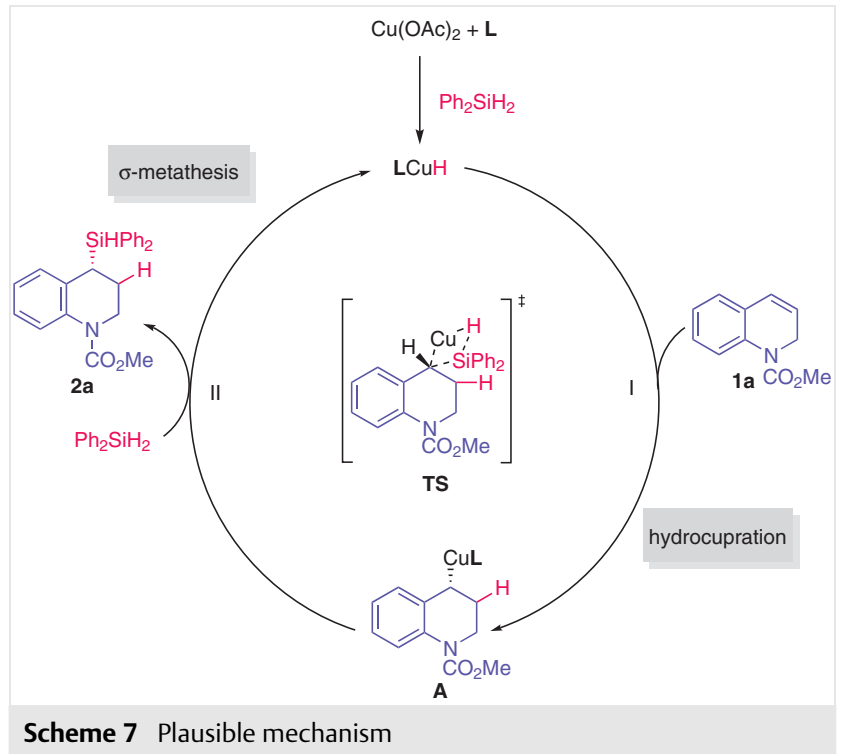

In conclusion, a copper(II) acetate $/(R, R)-\mathrm{Ph}-\mathrm{BPE} /(p-$ tolyl $)_{3} \mathrm{P}$ catalyzed asymmetric hydrosilylation of 1,2-dihydroquinolines with hydrosilanes was developed. Various 4silyl and 4-hydroxy 1,2,3,4-tetrahydroquinolines were obtained with good enantioselectivities. The enantioselective incorporation of a silyl group on the tetrahydroquinoline skeleton might find application in medicinal chemistry.

\section{Funding Information}

We thank MOST (2016YFA0202900), the NSFC (21821002, 21801248), the Chinese Academy of Sciences (XDB20030000, QYZDYSSW-SLH012), the Science and Technology Commission of Shanghai Municipality (18JC1411302, 18YF1428900, 19590750400) for generous financial support.

\section{Supporting Information}

Supporting information for this article is available online at https://doi.org/10.1055/s-0040-1707134.

\section{References and Notes}

(1) (a) Meanwell, N. A. J. Med. Chem. 2011, 54, 2529. (b) Lazareva, N. F.; Lazarev, I. M. Russ. Chem. Bull. 2015, 64, 1221. (c) Fujii, S.; Hashimoto, Y. Future Med. Chem. 2017, 9, 485. (d) Ramesh, R.; Reddy, D. S. J. Med. Chem. 2018, 61, 3779.

(2) (a) Mills, J. S.; Showell, G. A. Expert Opin. Invest. Drugs 2004, 13, 1149. (b) Showell, G. A.; Mills, J. S. Drug Discovery Today 2003, 8, 551. (c) Franz, A. K.; Wilson, S. O. J. Med. Chem. 2013, 56, 388.

(3) Fujii, S. MedChemComm 2016, 7, 1082.

(4) (a) Sieburth, S. McN.; Chen, C.-A. Eur. J. Org. Chem. 2006, 311. (b) Singh, S.; Sieburth, S. McN. Org. Lett. 2012, 14, 4422.

(5) Svennebring, A. J. Appl. Toxicol. 2016, 36, 483.

(6) For a review, see: (a) Gately, S.; West, R. Drug Dev. Res. 2007, 68, 156. For selected examples: (b) Van Hattum, A. H.; Pinedo, H. M.; Schlüper, H. M. M.; Hausheer, F. H.; Boven, E. Int. J. Cancer 2000, 88, 260. (c) Seetharamsingh, B.; Ramesh, R.; Dange, S. S.; Khairnar, P. V.; Singhal, S.; Upadhyay, D.; Veeraraghavan, S.; Viswanadha, S.; Vakkalanka, S.; Reddy, D. S. ACS Med. Chem. Lett. 2015, 6, 1105. (d) Jachak, G. R.; Ramesh, R.; Sant, D. G.; Jorwekar, S. U.; Jadhav, M. R.; Tupe, S. G.; Deshpande, M. V.; Reddy, D. S. ACS Med. Chem. Lett. 2015, 6, 1111. (e) Kajita, D.; Nakamura, M.; Matsumoto, Y.; Ishikawa, M.; Hashimoto, Y.; Fujii, S. Bioorg. Med. Chem. Lett. 2015, 25, 3350. (f) Luger, P.; Dittrich, B.; Tacke, R. Org. Biomol. Chem. 2015, 13, 9093. (g) Toyama, H.; Sato, S.; Shirakawa, H.; Komai, M.; Hashimoto, Y.; Fujii, S. Bioorg. Med. Chem. Lett. 2016, 26, 1817. (h) Ramesh, R.; Shingare, R. D.; Kumar, V.; Anand, A.; B, S.; Veeraraghavan, S.; Viswanadha, S.; Ummanni, R.; Gokhale, R.; Reddy, D. S. Eur. J. Med. Chem. 2016, 122, 723. (i) Panayides, J.-L.; Mathieu, V.; Banuls, L. M. Y.; Apostolellis, H.; Dahan-Farkas, N.; Davids, H.; Harmse, L.; Rey, M. E. C.; Green, I. R.; Pelly, S. C.; Kiss, R.; Kornienko, A.; van Otterlo, W. A. L. Bioorg. Med. Chem. 2016, 24, 2716.

(7) (a) Saito, K.; Kanai, M. Heterocycles 2012, 86, 1565. (b) Toutov, A. A.; Liu, W.-B.; Betz, K. N.; Stoltz, B. M.; Grubbs, R. H. Nat. Protoc. 2015, 10, 1897. (c) Toutov, A. A.; Liu, W.-B.; Betz, K. N.; Fedorov, A.; Stoltz, B. M.; Grubbs, R. H. Nature 2015, 518, 80. 
(8) (a) Vivet, B.; Cavelier, F.; Martinez, J. Eur. J. Org. Chem. 2000, 807. (b) Cavelier, F.; Vivet, B.; Martinez, J.; Aubry, A.; Didierjean, C.; Vicherat, A.; Marraud, M. J. Am. Chem. Soc. 2002, 124, 2917. (c) Cavelier, F.; Marchand, D.; Martinez, J.; Sagan, S. J. Pept. Res. 2004, 63, 290. (d) Pujals, S.; Fernández-Carneado, J.; Kogan, M. J.; Martinez, J.; Cavelier, F.; Giralt, E. J. Am. Chem. Soc. 2006, 128, 8479. (e) Hernández, D.; Nielsen, L.; Lindsay, K. B.; LópezGarcía, M. A.; Bjerglund, K.; Skrydstrup, T. Org. Lett. 2010, 12, 3528. (f) Wang, J.; Ma, C.; Wu, Y.; Lamb, R. A.; Pinto, L. H.; DeGrado, W. F. J. Am. Chem. Soc. 2011, 133, 13844. (g) Kim, J. K.; Sieburth, S. McN. J. Org. Chem. 2012, 77, 2901.

(9) (a) Mortensen, M.; Husmann, R.; Veri, E.; Bolm, C. Chem. Soc. Rev. 2009, 38, 1002. (b) Rémond, E.; Martin, C.; Martinez, J.; Cavelier, F. Chem. Rev. 2016, 116, 11654.

(10) (a) Katritzky, A. R.; Rachwal, S.; Rachwal, B. Tetrahedron 1996, 52, 15031. (b) Sridharan, V.; Suryavanshi, P. A.; Menéndez, J. C. Chem. Rev. 2011, 111, 7157. (c) Muñoz, G. D.; Dudley, G. B. Org. Prep. Proced. Int. 2015, 47, 179. (d) Muthukrishnan, I.; Sridharan, V.; Menéndez, J. C. Chem. Rev. 2019, 119, 5057.

(11) For selected reviews, see: (a) Masson, G.; Lalli, C.; Benohoud, M.; Dagousset, G. Chem. Soc. Rev. 2013, 42, 902. (b) Jiang, X.; Wang, R. Chem. Rev. 2013, 113, 5515. (c) Fochi, M.; Caruana, L.; Bernardi, L. Synthesis 2014, 46, 135. (d) Xie, M.; Lin, L.; Feng, X. Chem. Rec. 2017, 17, 1184. For selected recent examples, see: (e) Gelis, C.; Levitre, G.; Guérineau, V.; Touboul, D.; Neuville, L.; Masson, G. Eur. J. Org. Chem. 2019, 5151. (f) Kazancioglu, M. Z.; Kalay, E.; Kazancioglu, E. A.; Peshkov, V. A. ChemistrySelect 2019, 4, 8797. (g) Huang, J.-X.; Hou, K.-Q.; Hu, Q.-L.; Chen, X.-P.; Li, J.; Chan, A. S. C.; Xiong, X.-F. Org. Lett. 2020, 22, 1858. (h) Li, J.; Gu, Z.; Zhao, X.; Qiao, B.; Jiang, Z. Chem. Commun. 2019, 55, 12916. (i) Jarrige, L.; Gandon, V.; Masson, G. Chem. Eur. J. 2020, 26, 1406.

(12) For selected recent examples, see: (a) Mei, G.-J.; Li, D.; Zhou, G.X.; Shi, Q.; Cao, Z.; Shi, F. Chem. Commun. 2017, 53, 10030. (b) Tukhvatshin, R. S.; Kucherenko, A. S.; Nelyubina, Y. V.; Zlotin, S. G. Eur. J. Org. Chem. 2018, 7000. (c) Song, Y.-X.; Du, D.-M. Org. Biomol. Chem. 2018, 16, 9390. (d) Chen, J.; Han, X.; Lu, X. Org. Lett. 2019, 21, 8153.

(13) (a) Chen, L.; Zhang, L.; Lv, J.; Cheng, J.-P.; Luo, S. Chem. Eur. J. 2012, 18, 8891. (b) Suh, C. W.; Woo, S. B.; Kim, D. Y. Asian J. Org. Chem. 2014, 3, 399. (c) Cao, W.; Liu, X.; Guo, J.; Lin, L.; Feng, X. Chem. Eur. J. 2015, 21, 1632.

(14) For other selected recent cyclization reactions, see: (a) Xu, C.; Feng, Y.; Li, F.; Han, J.; He, Y.-M.; Fan, Q.-H. Organometallics 2019, 38, 3979. (b) Xiong, W.; Li, S.; Fu, B.; Wang, J.; Wang, Q.A.; Yang, W. Org. Lett. 2019, 21, 4173.

(15) (a) Levit, G. L.; Gruzdev, D. A.; Krasnov, V. P.; Chulakov, E. N.; Sadretdinova, L. Sh.; Ezhikova, M. A.; Kodess, M. I.; Charushin, V. N. Tetrahedron: Asymmetry 2011, 22, 185. (b) Gruzdev, D. A.; Chulakov, E. N.; Levit, G. L.; Ezhikova, M. A.; Kodess, M. I.; Krasnov, V. P. Tetrahedron: Asymmetry 2013, 24, 1240. (c) Qin, L.; Zheng, D.; Cui, B.; Wan, N.; Zhou, X.; Chen, Y. Tetrahedron Lett. 2016, 57, 2403.

(16) For selected reviews, see: (a) Wang, D.-S.; Chen, Q.-A.; Lu, S.-M.; Zhou, Y.-G. Chem. Rev. 2012, 112, 2557. (b) Zheng, C.; You, S.-L. Chem. Soc. Rev. 2012, 41, 2498. (c) Luo, Y.-E.; He, Y.-M.; Fan, Q.H. Chem. Rec. 2016, 16, 2697. (d) Meng, W.; Feng, X.; Du, H. Acc. Chem. Res. 2018, 51, 191. (e) Meng, W.; Feng, X.; Du, H. Chin. J. Chem. 2020, 38, 625. For selected recent examples, see: (f) Li, B.; Xu, C.; He, Y.-M.; Deng, G.-J.; Fan, Q.-H. Chin. J. Chem. 2018, 36, 1169. (g) Chen, Y.; He, Y.-M.; Zhang, S.; Miao, T.; Fan, Q.-H. Angew. Chem. Int. Ed. 2019, 58, 3809. (h) Hu, X.-H.; Hu, X.-P. Org. Lett. 2019, 21, 10003. (i) Liu, Y.; Chen, F.; He, Y.-M.; Li, C.; Fan,
Q.-H. Org. Biomol. Chem. 2019, 17, 5099. (j) Chen, Y.; Pan, Y.; He, Y.-M.; Fan, Q.-H. Angew. Chem. Int. Ed. 2019, 58, 16831. (k) Li, X.; Tian, J.-J.; Liu, N.; Tu, X.-S.; Zeng, N.-N.; Wang, X.-C. Angew. Chem. Int. Ed. 2019, 58, 4664. (1) Tao, L.; Li, C.; Ren, Y.; Li, H.; Chen, J.; Yang, Q. Chin. J. Catal. 2019, 40, 1548. (m) Tao, L.; Ren, Y.; Li, C.; Li, H.; Chen, X.; Liu, L.; Yang, Q. ACS Catal. 2020, 10, 1783. (n) Wang, L.-R.; Chang, D.; Feng, Y.; He, Y.-M.; Deng, G.-J.; Fan, Q.-H. Org. Lett. 2020, 22, 2251.

(17) For selected recent examples, see: (a) Fischer, T.; Duong, Q.-N.; Mancheño, O. G. Chem. Eur. J. 2017, 23, 5983. (b) Duong, Q.-N.; Schifferer, L.; Mancheño, O. G. Eur. J. Org. Chem. 2019, 5452.

(18) (a) Li, G.; Liu, H.; Wang, Y.; Zhang, S.; Lai, S.; Tang, L.; Zhao, J.; Tang, Z. Chem. Commun. 2016, 52, 2304. (b) Kubota, K.; Watanabe, Y.; Ito, H. Adv. Synth. Catal. 2016, 358, 2379. (c) Kong, D.; Han, S.; Wang, R.; Li, M.; Zi, G.; Hou, G. Chem. Sci. 2017, 8, 4558. (d) Kong, D.; Han, S.; Zi, G.; Hou, G.; Zhang, J. J. Org. Chem. 2018, 83, 1924. (e) Xu-Xu, Q.-F.; Zhang, X.; You, S.-L. Org. Lett. 2019, 21, 5357. (f) Xu-Xu, Q.-F.; Zhang, X.; You, S.-L. Org. Lett. 2020, 22, 1530. (g) For a related example, see: Kubota, K.; Watanabe, Y.; Hayama, K.; Ito, H. J. Am. Chem. Soc. 2016, 138, 4338.

(19) (a) Lukevics, E.; Germane, S.; Segal, I.; Zablotskaya, A. Chem. Heterocycl. Compd. 1997, 33, 234. (b) Gandhamsetty, N.; Joung, S.; Park, S.-W.; Park, S.; Chang, S. J. Am. Chem. Soc. 2014, 136, 16780.

(20) For selected reviews, see: (a) Park, S. Chin. J. Chem. 2019, 37, 1057. (b) Wilkinson, J. R.; Nuyen, C. E.; Carpenter, T. S.; Harruff, S. R.; Hoveln, R. V. ACS Catal. 2019, 9, 8961. For selected recent examples, see: (c) Kan, S. B. J.; Lewis, R. D.; Chen, K.; Arnold, F. H. Science 2016, 354, 1048. (d) Wang, A.; Bernasconi, M.; Pfaltz, A. Adv. Synth. Catal. 2017, 359, 2523. (e) Zuo, Z.; Xu, S.; Zhang, L.; Gan, L.; Fang, H.; Liu, G.; Huang, Z. Organometallics 2019, 38, 3906. (f) Chowdhury, R.; Dubey, A. K.; Ghosh, S. K. J. Org. Chem. 2019, 84, 2404.

(21) For selected reviews, see: (a) Han, J. W.; Hayashi, T. Tetrahedron: Asymmetry 2014, 25, 479. (b) Chen, J.; Guo, J.; Lu, Z. Chin. J. Chem. 2018, 36, 1075. (c) Chen, J.; Lu, Z. Org. Chem. Front. 2018, 5, 260. (d) Zaranek, M.; Pawluc, P. ACS Catal. 2018, 8, 9865. For selected examples, see: (e) Yamamoto, K.; Hayashi, T.; Kumada, M. J. Am. Chem. Soc. 1971, 93, 5301. (f) Kiso, Y.; Yamamoto, K.; Tamao, K.; Kumada, M. J. Am. Chem. Soc. 1972, 94, 4373. (g) Uozumi, Y.; Hayashi, T. J. Am. Chem. Soc. 1991, 113, 9887. (h) Tamao, K.; Nakamura, K.; Ishii, H.; Yamaguchi, S.; Shiro, M. J. Am. Chem. Soc. 1996, 118, 12469. (i) Jensen, J. F.; Svendsen, B. Y.; la Cour, T. V.; Pedersen, H. L.; Johannsen, M. J. Am. Chem. Soc. 2002, 124, 4558. (j) Naito, T.; Yoneda, T.; Ito, J.-i.; Nishiyama, H. Synlett 2012, 23, 2957. (k) Chen, J.; Cheng, B.; Cao, M.; Lu, Z. Angew. Chem. Int. Ed. 2015, 54, 4661. (l) Cheng, B.; Lu, P.; Zhang, H.; Cheng, X.; Lu, Z. J. Am. Chem. Soc. 2017, 139, 9439. (m) Zhao, Z.-Y.; Nie, Y.-X.; Tang, R.-H.; Yin, G.-W.; Cao, J.; Xu, Z.; Cui, Y.-M.; Zheng, Z.-J.; Xu, L.-W. ACS Catal. 2019, 9, 9110.

(22) (a) Trost, B. M.; Dong, G. J. Am. Chem. Soc. 2010, 132, 16403. (b) Trost, B. M.; Frontier, A. J.; Thiel, O. R.; Yang, H.; Dong, G. Chem. Eur. J. 2011, 17, 9762. (c) Trost, B. M.; Yang, H.; Brindle, C. S.; Dong, G. Chem. Eur. J. 2011, 17, 9777.

(23) For selected asymmetric examples, see: (a) Gribble, M. W., Jr.; Pirnot, M. T.; Bandar, J. S.; Liu, R. Y.; Buchwald, S. L. J. Am. Chem. Soc. 2017, 139, 2192. (b) Zhang, L.; Oestreich, M. Chem. Eur. J. 2019, 25, 14304. (c) Mao, W.; Xue, W.; Irran, E.; Oestreich, M. Angew. Chem. Int. Ed. 2019, 58, 10723. For selected racemic versions, see: (d) Zhao, X.; Xu, S.; He, J.; Zhou, Y.; Cao, S. Org. Chem. Front. 2019, 6, 2539. (e) Wang, H.; Zhang, G.; Zhang, Q.; Wang, Y.; Li, Y.; Xiong, T.; Zhang, Q. Chem. Commun. 2020, 56, 1819. 
(24) Lipshutz, B. H.; Noson, K.; Chrisman, W.; Lower, A. J. Am. Chem. Soc. 2003, 125, 8779.

(25) Methyl (R)-4-(Diphenylsilyl)-3,4-dihydroquinoline-1(2H)carboxylate (2a): Yield: $64.1 \mathrm{mg}(86 \%)$; colorless oil; $87 \%$ ee [Daicel Chiralpak OD-H $(0.46 \mathrm{~cm} \times 25 \mathrm{~cm}), n$-hexane/2-propa$\mathrm{nol}=90 / 10, v=0.7 \mathrm{~mL} \cdot \mathrm{min}^{-1}, \lambda=230 \mathrm{~nm}, t_{\mathrm{R}}$ (major) $=12.59$ $\min , t_{\mathrm{R}}($ minor $\left.)=10.58 \mathrm{~min}\right] ;[\alpha]_{\mathrm{D}}{ }^{28}=+18.9\left(c=1.0, \mathrm{CHCl}_{3}\right) .{ }^{1} \mathrm{H}$ NMR $\left(400 \mathrm{MHz}, \mathrm{CDCl}_{3}\right): \delta=7.65-7.50(\mathrm{~m}, 1 \mathrm{H}), 7.50-7.44(\mathrm{~m}$, $2 \mathrm{H}), 7.44-7.35(\mathrm{~m}, 4 \mathrm{H}), 7.35-7.26(\mathrm{~m}, 4 \mathrm{H}), 7.12-7.05(\mathrm{~m}, 1 \mathrm{H})$, 6.92-6.85 (m, $2 \mathrm{H}), 4.92(\mathrm{~d}, J=2.8 \mathrm{~Hz}, 1 \mathrm{H}), 3.93(\mathrm{dt}, J=12.4$, $6.4 \mathrm{~Hz}, 1 \mathrm{H}), 3.65(\mathrm{~s}, 3 \mathrm{H}), 3.31(\mathrm{dt}, J=12.0,6.0 \mathrm{~Hz}, 1 \mathrm{H}), 2.99(\mathrm{td}$, $J=6.8,3.2 \mathrm{~Hz}, 1 \mathrm{H}), 2.24-2.06(\mathrm{~m}, 2 \mathrm{H}) .{ }^{13} \mathrm{C}$ NMR $(100 \mathrm{MHz}$, $\left.\mathrm{CDCl}_{3}\right): \delta=155.1,138.1,135.7,135.6,132.6,132.5,131.7,130.0$, $129.9,128.5,128.2,128.1,125.2,124.7,123.8,52.8,44.2,26.2$, 25.7. IR (thin film): 3057, 3010, 2945, 2123, 1699, 1587, 1487, $1435,1377,1329,1249,1193,1113,1048,803,736,698,582$, $481,434 \mathrm{~cm}^{-1}$. HRMS (ESI): $\mathrm{m} / z[\mathrm{M}+\mathrm{Na}]^{+}$calcd for $\mathrm{C}_{23} \mathrm{H}_{23} \mathrm{NN}-$ $\mathrm{aO}_{2} \mathrm{Si}$ : 396.1390; found: 396.1390 .

(26) General Procedure A: An oven-dried $10 \mathrm{~mL}$ screw-cap reaction tube with magnetic stir bar was charged with copper acetate (1.8 mg, $0.010 \mathrm{mmol}, 5.0 \mathrm{~mol} \%),(R, R)$-Ph-BPE (5.6 mg, 0.011 $\mathrm{mmol}, 5.5 \mathrm{~mol} \%)$ and tri(p-tolyl)phosphine $(6.7 \mathrm{mg}, 0.022$ mmol, $11 \mathrm{~mol} \%)$. It was evacuated and backfilled with argon three times. Diphenylsilane ( $111 \mu \mathrm{L}, 0.6 \mathrm{mmol})$ was added by using a syringe and the resulting mixture was premixed for 30 min at $30^{\circ} \mathrm{C}$ on a heating block. To the resulting orange mixture, 1,2-dihydroquinoline $\mathbf{1}(0.2 \mathrm{mmol})$ was added under argon atmosphere. The mixture was stirred for $36 \mathrm{~h}$ at $40{ }^{\circ} \mathrm{C}$ on a heating block. The mixture was diluted with ethyl acetate $(20$ $\mathrm{mL}$ ), then the organic phase was allowed to pass through a short pad of silica gel with extra ethyl acetate $(20 \mathrm{~mL})$ as eluent. The filtrate was concentrated in vacuo and the crude mixture was purified by silica gel column chromatography $(\mathrm{PE} / \mathrm{EtOAC}=100: 1$ to $40: 1, \mathrm{v} / \mathrm{v})$ or preparative TLC $(\mathrm{PE} / \mathrm{EtOAC}=40: 1, \mathrm{v} / \mathrm{v})$ affording product 2.

(27) General Procedure B: An oven-dried $10 \mathrm{~mL}$ screw-cap reaction tube with magnetic stir bar was charged with copper acetate (1.8 mg, $0.010 \mathrm{mmol}, 5.0 \mathrm{~mol} \%),(R, R)$-Ph-BPE (5.6 mg, 0.011 mmol, $5.5 \mathrm{~mol} \%)$ and tri(p-tolyl)phosphine $(6.7 \mathrm{mg}, 0.022$ $\mathrm{mmol}, 11 \mathrm{~mol} \%$ ). It was evacuated and backfilled with argon for three times. Phenylsilane ( $74 \mu \mathrm{L}, 0.6 \mathrm{mmol}$ ) was added by using a syringe and the resulting mixture was premixed at r.t. for 5 min. To the resulting orange mixture, 1,2-dihydroquinoline $\mathbf{1}$ $(0.2 \mathrm{mmol})$ was added under argon atmosphere. The mixture was stirred for $36 \mathrm{~h}$ at $40{ }^{\circ} \mathrm{C}$ on a heating block. The volatiles were removed in vacuo with an oil pump at room temperature and the crude product was used for the next step without further purification. To a $25 \mathrm{~mL}$ Schlenk tube were added potassium fluoride (46.5 mg, $0.8 \mathrm{mmol}), \mathrm{K}_{2}$ EDTA $\left(\mathrm{H}_{2} \mathrm{O}\right)_{2}(80.9 \mathrm{mg}, 0.2$ $\mathrm{mmol}$ ) and potassium bicarbonate ( $80.1 \mathrm{mg}, 0.8 \mathrm{mmol})$ and the tube was evacuated and backfilled with argon for three times. The crude product was dissolved in THF $(1 \mathrm{~mL})$ and transferred to the Schlenk tube by using a syringe. The residue was further rinsed with THF $(0.1 \mathrm{~mL} \times 2)$ and added to the tube. To the resulting mixture was added methanol $(1.2 \mathrm{~mL})$ dropwise and gas was released. The mixture was stirred at room temperature for $40 \mathrm{~min}$, then hydrogen peroxide $(0.23 \mathrm{~g}, 27 \% \mathrm{w} / \mathrm{w}$ in water, $1.8 \mathrm{mmol}$ ) was added and the suspension was stirred at room temperature for $20 \mathrm{~h}$. The reaction was quenched with sodium thiosulfate $(0.85 \mathrm{~g}, 5.4 \mathrm{mmol})$ with extra methanol $(2 \mathrm{~mL})$. After peroxide residue was quenched completely as indicated by starch-iodine indicator paper, the mixture was diluted by ethyl acetate $(5 \mathrm{~mL})$, dried over magnesium sulfate, filtered by glasssintered filter, rinsed with extra ethyl acetate $(20 \mathrm{~mL})$ and concentrated in vacuo. The crude product was purified by silica gel column chromatography $(\mathrm{PE} / \mathrm{EtOAc}=10: 1$ to $2: 1, \mathrm{v} / \mathrm{v})$ or preparative TLC $(\mathrm{PE} / \mathrm{EtOAC}=2: 1, \mathrm{v} / \mathrm{v})$ afford product 3 .

(28) Methyl (R)-4-Hydroxy-3,4-dihydroquinoline-1(2H)-carboxylate (3a): Yield: $26.0 \mathrm{mg}$ (63\%); colorless oil; $89 \%$ ee [Daicel Chiralpak IG $(0.46 \mathrm{~cm} \times 25 \mathrm{~cm})$, $n$-hexane/2-propanol = 95:5, $v=$ $1.0 \mathrm{~mL} \cdot \mathrm{min}^{-1}, \lambda=230 \mathrm{~nm}, t_{\mathrm{R}}$ (major) $=36.31 \mathrm{~min}, t_{\mathrm{R}}($ minor $)=$ $33.64 \mathrm{~min}] ;[\alpha]_{\mathrm{D}}{ }^{21}=+25.6\left(c=1.0, \mathrm{CHCl}_{3}\right) .{ }^{1} \mathrm{H} \operatorname{NMR}(400 \mathrm{MHz}$, $\left.\mathrm{CDCl}_{3}\right): \delta=7.80(\mathrm{~d}, J=8.4 \mathrm{~Hz}, 1 \mathrm{H}), 7.38(\mathrm{~d}, J=7.6 \mathrm{~Hz}, 1 \mathrm{H}), 7.26$ $(\mathrm{t}, J=8.0 \mathrm{~Hz}, 1 \mathrm{H}), 7.09(\mathrm{t}, J=7.6 \mathrm{~Hz}, 1 \mathrm{H}), 4.80-4.70(\mathrm{~m}, 1 \mathrm{H})$, $4.07(\mathrm{dt}, J=13.2,5.2 \mathrm{~Hz}, 1 \mathrm{H}), 3.79(\mathrm{~s}, 3 \mathrm{H}), 3.64$ (ddd, $J=13.6$, 10.0, $4.4 \mathrm{~Hz}, 1 \mathrm{H}), 2.18(\mathrm{~s}, 1 \mathrm{H}), 2.14-1.93(\mathrm{~m}, 2 \mathrm{H}) .{ }^{13} \mathrm{C}$ NMR $\left(100 \mathrm{MHz}, \mathrm{CDCl}_{3}\right): \delta=155.1,137.4,130.8,128.4,128.3,124.0$, 123.4, 65.8, 53.1, 40.7, 31.9. IR (thin film): 3399, 2953, 1681, $1605,1581,1490,1439,1377,1331,1245,1217,1192,1134$, 1083, 1054, 1037, 1021, 976, 943, 911, 865, 821, 756, 702, 591, 560, 530, $491 \mathrm{~cm}^{-1}$. HRMS (ESI): $\mathrm{m} / z[\mathrm{M}+\mathrm{Na}]^{+}$calcd for $\mathrm{C}_{11} \mathrm{H}_{13} \mathrm{NNaO}_{3}$ : 230.0788; found: 230.0791 .

(29) CCDC 1996641 contains the supplementary crystallographic data for compound $\mathbf{4}$. The data can be obtained free of charge from The Cambridge Crystallographic Data Centre via www.ccdc.cam.ac.uk/getstructures.

(30) (a) Fu, P.-F.; Brard, L.; Li, Y.; Marks, T. J. J. Am. Chem. Soc. 1995, 117, 7157. (b) Chen, Y.; Sui-Seng, C.; Boucher, S.; Zargarian, D. Organometallics 2005, 24, 149. (c) Noh, D.; Chea, H.; Ju, J.; Yun, J. Angew. Chem. Int. Ed. 2009, 48, 6062. (d) Noh, D.; Yoon, S. K.; Won, J.; Lee, J. Y.; Yun, J. Chem. Asian J. 2011, 6, 1967. (e) Xi, Y.; Hartwig, J. F. J. Am. Chem. Soc. 2017, 139, 12758. 